Nuclear Instruments and Methods 172 (1980) 439-446

(c) North-Holland Publishing Company

\title{
IMPROVEMENT OF THE PERFORMANCE OF A COMPTON-SUPPRESSION SPECTROMETER BY MINIMIZING THE DEAD LAYER OF THE CENTRAL Ge DETECTOR
}

\author{
H.J.M. AARTS, G.A.P. ENGELBERTINK, C.J. VAN DER POEL, D.E.C. SCHERPENZEEL and H.F.R. \\ ARCISZEWSKI \\ Fysisch Laboratorium, Rijksuniversiteit Utrecht, Princetonplein 5, 3508 TA Utrecht, The Netherlands
}

Received 12 December 1979

The performance of a Compton-suppression spectrometer is investigated for two different $\mathrm{Ge}$ crystals as central detector; a $126 \mathrm{~cm}^{3} \mathrm{Ge}(\mathrm{Li})$ with a dead layer of $1.0 \mathrm{~mm}$ and a $90 \mathrm{~cm}^{3} \mathrm{HPGe}$ with a dead layer of $0.22 \mathrm{~mm}$. The thin dead layer HPGe gives a 32\% improvement in the overall Compton suppression. Predictions of the influence of the dead-layer thickness by means of Monte Carlo calculations are in good agreement. The spectrometer is further tested with radioactive sources of ${ }^{88} \mathrm{Y}$ and ${ }^{152}$ Eu and the in-beam reaction ${ }^{24} \mathrm{Mg}+(45 \mathrm{MeV})^{16} \mathrm{O}$.

\section{Introduction}

During the last few years there has been an increasing interest in the use of Compton-suppression spectrometers for in-beam $\gamma$-ray spectroscopy with heavy-ion induced reactions, since the $\gamma$-ray spectra produced in these reactions are rather complex and therefore demand spectrometers with high resolution and low background.

In a Compton-suppression spectrometer (CSS) the background, which normally almost completely originates from Compton-scattered $\gamma$-rays, is reduced by surrounding the primary Ge detector with a scintillator, like $\mathrm{NaI}(\mathrm{Tl})$ or plastic. Only events in the central detector which occur without simultaneous observation of scattered quanta in the surrounding shield are accepted.

The inherent lower absolute efficiency of such a spectrometer, due to the required larger minimum distance between the target and central detector, would generally be a serious disadvantage for in-beam $\gamma$-ray spectroscopy. However, heavy-ion induced reactions show a reasonably large $\gamma$-ray yield and a large solid angle is not always very desirable in view of the Doppler broadening of the peaks. In practice the lower efficiency can be compensated for by the use of a higher beam intensity.

The actual performance of a CSS is, apart from the dimensions of the anticoincidence shield, mainly determined by the quality of the central detector and the amount of non-detecting material between the central detector and the surrounding shield. As far as the transmission is concerned the dead $\mathrm{Ge}$ layer around the central Ge crystal is the main source of non-detecting material and in the present paper its effect on the background is investigated by comparing CSS performance for a conventional $\mathrm{Ge}(\mathrm{Li})$ crystal with a $1.0 \mathrm{~mm}$ dead layer and a highpurity germanium (HPGe) crystal with a $0.2 \mathrm{~mm}$ thick Li-diffused outer contact.

To investigate the effect of a continuous variation of the thickness of the dead layer, Monte Carlo calculations have been performed.

\section{Monte Carlo calculations}

A Monte Carlo program has been written to simulate the histories of photons in a CSS configuration shown in fig. 1. A $115 \mathrm{~cm}^{3}$ cylindrical closedend $\mathrm{Ge}$ crystal with a dead core as an inner contact and a dead layer of variable thickness on the outside is used as a central detector. The Ge crystal is surrounded by a $1.0 \mathrm{~mm}$ thick aluminium cap. The influence of the $\mathrm{MgO}$ reflector and of the aluminium cover of the $\mathrm{NaI}$ can be neglected in the calculations since their contribution is relatively small. Multiple processes and an appropriate angular distribution for Compton scattering [1] are taken into account. It is assumed that in the absorption processes no second- 
Nuclear Instruments and Methods 172 (1980) 439-446

(c) North-Holland Publishing Company

\title{
IMPROVEMENT OF THE PERFORMANCE OF A COMPTON-SUPPRESSION SPECTROMETER BY MINIMIZING THE DEAD LAYER OF THE CENTRAL Ge DETECTOR
}

\author{
H.J.M. AARTS, G.A.P. ENGELBERTINK, C.J. VAN DER POEL, D.E.C. SCHERPENZEEL and H.F.R. \\ ARCISZEWSKI \\ Fysisch Laboratorium, Rijksuniversiteit Utrecht, Princetonplein 5, 3508 TA Utrecht, The Netherlands
}

Received 12 December 1979

The performance of a Compton-suppression spectrometer is investigated for two different Ge crystals as central detector; a $126 \mathrm{~cm}^{3} \mathrm{Ge}(\mathrm{Li})$ with a dead layer of $1.0 \mathrm{~mm}$ and a $90 \mathrm{~cm}^{3} \mathrm{HPGe}$ with a dead layer of $0.22 \mathrm{~mm}$. The thin dead layer HPGe gives a 32\% improvement in the overall Compton suppression. Predictions of the influence of the dead-layer thickness by means of Monte Carlo calculations are in good agreement. The spectrometer is further tested with radioactive sources of ${ }^{88} \mathrm{Y}$ and ${ }^{152} \mathrm{Eu}$ and the in-beam reaction ${ }^{24} \mathrm{Mg}+(45 \mathrm{MeV})^{16} \mathrm{O}$.

\section{Introduction}

During the last few years there has been an increasing interest in the use of Compton-suppression spectrometers for in-beam $\gamma$-ray spectroscopy with heavy-ion induced reactions, since the $\gamma$-ray spectra produced in these reactions are rather complex and therefore demand spectrometers with high resolution and low background.

In a Compton-suppression spectrometer (CSS) the background, which normally almost completely originates from Compton-scattered $\gamma$-rays, is reduced by surrounding the primary $\mathrm{Ge}$ detector with a scintillator, like $\mathrm{NaI}(\mathrm{Tl})$ or plastic. Only events in the central detector which occur without simultaneous observation of scattered quanta in the surrounding shield are accepted.

The inherent lower absolute efficiency of such a spectrometer, due to the required larger minimum distance between the target and central detector, would generally be a serious disadvantage for in-beam $\gamma$-ray spectroscopy. However, heavy-ion induced reactions show a reasonably large $\gamma$-ray yield and a large solid angle is not always very desirable in view of the Doppler broadening of the peaks. In practice the lower efficiency can be compensated for by the use of a higher beam intensity.

The actual performance of a CSS is, apart from the dimensions of the anticoincidence shield, mainly determined by the quality of the central detector and the amount of non-detecting material between the central detector and the surrounding shield. As far as the transmission is concerned the dead $\mathrm{Ge}$ layer around the central Ge crystal is the main source of non-detecting material and in the present paper its effect on the background is investigated by comparing CSS performance for a conventional $\mathrm{Ge}(\mathrm{Li})$ crystal with a $1.0 \mathrm{~mm}$ dead layer and a highpurity germanium (HPGe) crystal with a $0.2 \mathrm{~mm}$ thick Li-diffused outer contact.

To investigate the effect of a continuous variation of the thickness of the dead layer, Monte Carlo calculations have been performed.

\section{Monte Carlo calculations}

A Monte Carlo program has been written to simulate the histories of photons in a CSS configuration shown in fig. 1 . A $115 \mathrm{~cm}^{3}$ cylindrical closedend $\mathrm{Ge}$ crystal with a dead core as an inner contact and a dead layer of variable thickness on the outside is used as a central detector. The Ge crystal is surrounded by a $1.0 \mathrm{~mm}$ thick aluminium cap. The influence of the $\mathrm{MgO}$ reflector and of the aluminium cover of the $\mathrm{NaI}$ can be neglected in the calculations since their contribution is relatively small. Multiple processes and an appropriate angular distribution for Compton scattering [1] are taken into account. It is assumed that in the absorption processes no second- 


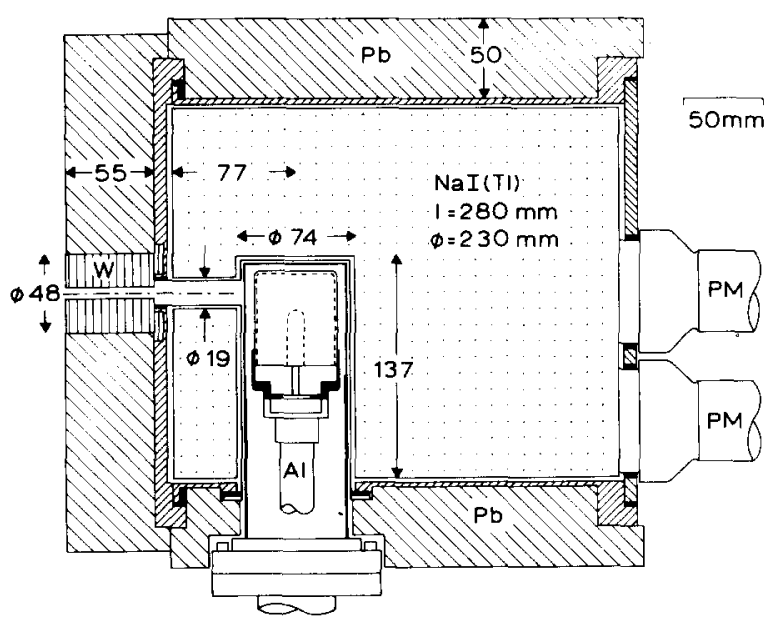

Fig. 1. Schematic drawing of the Compton-suppression spectrometer. The $\gamma$-rays enter the system through a collimator at the left hand side. At the right hand side two of the six multipliers are indicated. All dimensions are in $\mathrm{mm}$.

ary electrons or bremsstrahlung escape from the central crystal, so that the validity of the calculations is restricted to not too small Ge volumes and not too high-energy $\gamma$-rays $\left(E_{\gamma} \leqslant 3 \mathrm{MeV}\right)$,

In the Monte Carlo calculations spectra of ${ }^{60} \mathrm{Co}$ are generated. To obtain a spectrum, $10^{5}$ photons for each energy (1.17 and $1.33 \mathrm{MeV})$ are emitted from a point source in front of the collimator. The signal from the central detector is vetoed when the energy

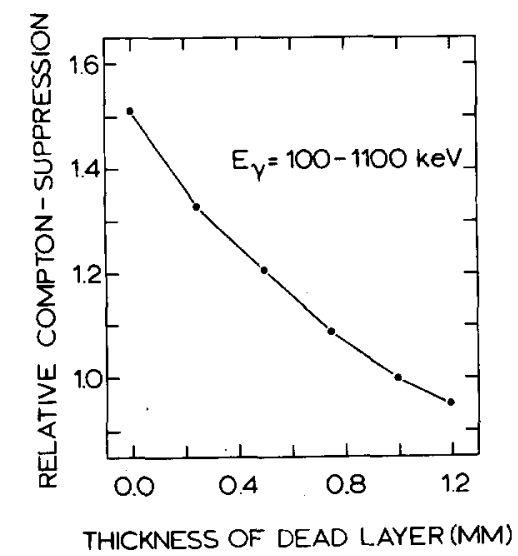

Fig. 2. Result of the Monte Carlo calculation for the relative Compton suppression as a function of the thickness of the dead layer of the central detector. The curve is normalized to unity for a dead-leayer thickness of $1.0 \mathrm{~mm}$. The statistical errors are smaller than the points. absorbed in the NaI shield exceeds a lower level of $50 \mathrm{keV}$.

In fig. 2 we show the calculated Compton suppression averaged over the energy region 100-1100 $\mathrm{keV}$. The curve is relative and normalized to unity for a dead layer of $1.0 \mathrm{~mm}$.

For a reduction of the dead layer from 1.0-0.22 $\mathrm{mm}$, the Monte Carlo calculation predicts an improvement of about $35 \%$ for the overall Compton suppression.

In the next section the Monte Carlo prediction is compared with test measurements.

\section{Experimental results}

The geometry of the NaI(Tl) anticoincidence shield is shown in fig. 1. The shield has been used in studies of high-spin states in the sd shell as reported in ref. [2].

Two crystals have been compared as central detectors; a $126 \mathrm{~cm}^{3} \mathrm{Ge}(\mathrm{Li})$ crystal with a dead layer of $1.0 \mathrm{~mm}$ and a dead core and a $90 \mathrm{~cm}^{3}$ HPGe crystal with a dead layer of $0.22 \mathrm{~mm}$ and a hollow core. More specific properties of both detectors are given in table 1 .

Table 1

Properties of the two detectors used as central crystal in a CSS

\begin{tabular}{lll}
\hline Manufacturer & $\begin{array}{l}\text { Philips } \\
\text { Nederland BV }\end{array}$ & $\begin{array}{l}\text { Harshaw Chemie BV } \\
\text { Holland }\end{array}$ \\
Type & Ge(Li) & HPGe \\
Active volume & $126 \mathrm{~cm}^{3}$ & $90 \mathrm{~cm}^{3}$ \\
Core & Inactive germanium & Hollow \\
Thickness & $1.0 \mathrm{~mm}$ & $0.22 \mathrm{~mm}$ \\
lead layer & & \\
$\begin{array}{l}\text { Thickness } \\
\text { Al-cap: front }\end{array}$ & $0.5 \mathrm{~mm}$ & $0.5 \mathrm{~mm}$ \\
$\quad$ side & $0.5 \mathrm{~mm}$ & $0.8 \mathrm{~mm}$ \\
$\begin{array}{l}\text { Operating } \\
\text { voltage }\end{array}$ & $4500 \mathrm{~V}$ & $3000 \mathrm{~V}$ \\
$\begin{array}{l}\text { Efficiency } \\
\text { fwhm }\end{array}$ & $27 \%$ & $20 \%$ \\
(1.33 MeV, & $2.4 \mathrm{keV}$ & $1.9 \mathrm{keV}$ \\
60 Co) & & 50 \\
$\begin{array}{l}\text { Peak-to- } \\
\text { Compton ratio }\end{array}$ & 46 & \\
\hline
\end{tabular}




\subsection{The thickness of the dead layer of the HPGe detector}

The thickness of the dead layer of the HPGe detector was obtained from the observed area ratio of the $(79.6+81.0) \mathrm{keV}$ complex and the $53.1 \mathrm{keV}$ $\gamma$-rays from a collimated ${ }^{133} \mathrm{Ba}$ source, in the way as shown in fig. 3.

With this method the thickness of the dead layer is given by

$d_{\mathrm{Ge}}=\left[\ln \left(\frac{A_{(80+81)}}{A_{53}}\right)-\ln \left(\frac{I_{(80+81)}}{I_{53}}\right)-p d_{\mathrm{Al}}\right] / q$

with $d_{\mathrm{Ge}}=$ the thickness of the $\mathrm{Ge}$ dead layer in $\mathrm{cm}$, $A_{(80+81)} / A_{53}=$ the observed ratio of the area of both the $(80+81) \mathrm{keV}$ and $53 \mathrm{keV}$ peaks, $I_{(80+81)} / I_{53}=$ ratio of the intensities of the $(80+81) \mathrm{keV}$ and 53 $\mathrm{keV}$ incoming radiation,

$p=\mu_{\mathrm{Al}}(53 \mathrm{keV})-\mu_{\mathrm{Al}}(81 \mathrm{keV})=0.376 \mathrm{~cm}^{-1}$

(the difference of the linear attenuation coefficients for aluminium, see ref. [3]), $d_{\mathrm{Al}}=$ the thickness of the aluminium can in $\mathrm{cm}$ and

$q=\mu_{\mathrm{Ge}}(53 \mathrm{keV})-\mu_{\mathrm{Ge}}(81 \mathrm{keV})=10.62 \mathrm{~cm}^{-1}$

(ref. [3]). The term $p d_{\mathrm{Al}}$ in eq. (1) is practically negligible for the present case with $d_{\mathrm{Al}}=0.08 \mathrm{~cm}$.

The intensity ratio $I_{(80+81)} / I_{53}^{*}$ is given by Gehrke et al. [4] as $14.8 \pm 1.1$.

Because this value is rather inaccurate, the intensity ratio has been measured with a $5 \mathrm{~cm}^{3}$ lowenergy photon spectrometer (LEPS) with a $250 \mu \mathrm{m}$ thick Be entrance window, an ion-implanted $0.3 \mu \mathrm{m}$ thick front contact and $10 \mathrm{~mm}$ depletion depth. The data are given in fig. 4 . They lead to $I_{(80+81)} / I_{53}=$ $16.6 \pm 0.2$. Corrections due to self-absorption in the source, entrance window, front contact and depletion depth are taken into account. Their combined influence is less than $1 \%$. Measurements of the irradiation at different angles also give consistent results.

With $I_{(80+81)} / I_{53}=16.6 \pm 0.2$ and the present $d_{\mathrm{Al}}=0.08 \mathrm{~cm}$, eq. (1), reduces to

$d_{\mathrm{Ge}}=\left[\ln \left(\frac{A_{(80+81)}}{A_{53}}\right)-(2.839 \pm 0.012)\right]$

$\times 0.0942 \mathrm{~cm}$.

This relationship is depicted by the curve in the lower part of fig. 3 .

The measured area ratio $A_{(80+81)} / A_{53}=21.5 \pm 0.2$
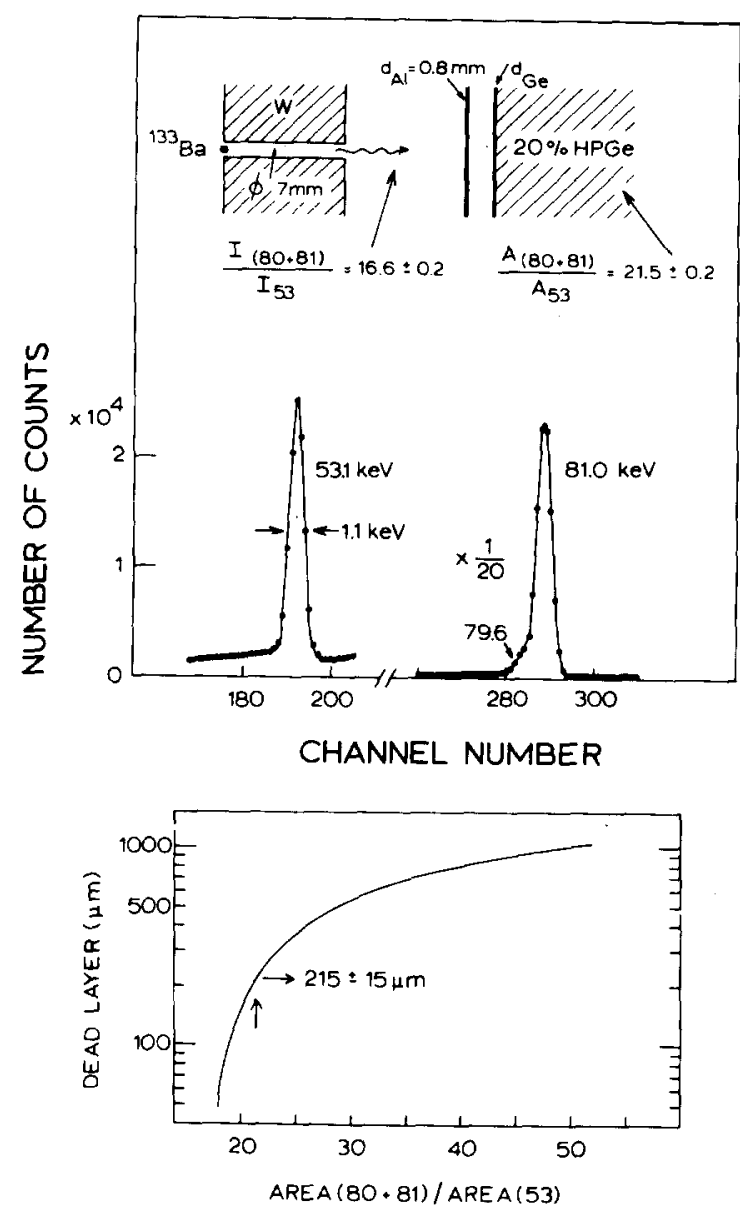

Fig. 3. Method to measure the dead-layer thickness of a $\mathrm{Ge}$ detector. The method is based on the difference in transmission of the dead layer for the $(80+81) \mathrm{keV}$ complex and the $53 \mathrm{keV} \gamma$-ray of a ${ }^{133} \mathrm{Ba}$ source. For the present $\mathrm{HPGe}$ detector the area ratio amounts to $A(80+81) / A_{53}=21.5 \pm$ 0.2 . The lower part gives the thickness of the dead layer as a function of the measured area ratio.

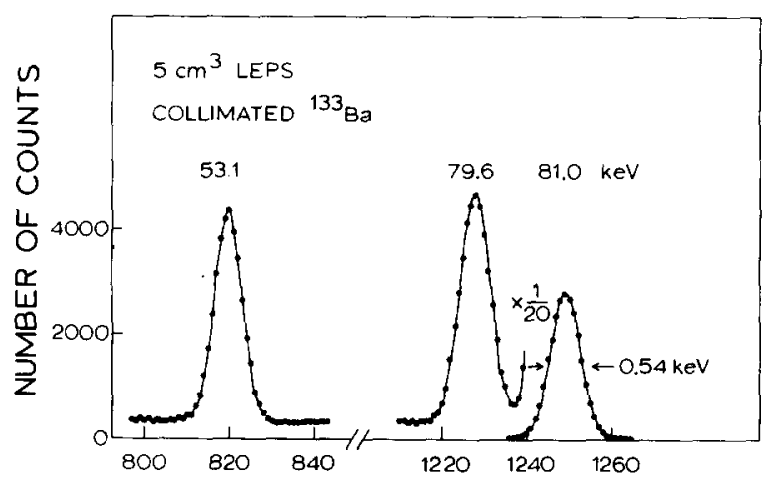

CHANNEL NUMBER

Fig. 4. The $53.1,79.6$ and $81.0 \mathrm{keV} \gamma$-rays from ${ }^{133} \mathrm{Ba}$ as measured with a $5 \mathrm{~cm}^{3}$ LEPS. 
implies a thickness of $(215 \pm 15) \mu \mathrm{m}$ for the dead layer of the HPGe detector.

For the $126 \mathrm{~cm}^{3} \mathrm{Ge}(\mathrm{Li})$, the area ratio is measured as $48.3 \pm 1.1$, which corresponds to a deadlayer thickness of $(980 \pm 25) \mu \mathrm{m}$.

From the data of fig. 4 follows as a byproduct $I_{81.0} / I_{79.6}=12.5 \pm 0.2$ for the intensity ratio of the 81.0 and $79.6 \mathrm{keV} \gamma$-rays from ${ }^{133} \mathrm{Ba}$.

\subsection{Experimental performance of the CSS for two different crystals as the central detector}

With the HPGe crystal as the central detector a ${ }^{60} \mathrm{Co}$ spectrum has been recorded with a PDP $11 / 40$ computer. The HPGe-NaI anti-coincidence pulse is used to gate two ADCs, such that two spectra, one coincident and one anti-coincident with the $\mathrm{NaI}$ pulse, are recorded simultaneously. The results are shown in fig. 5 . The upper spectrum is the sum of the two recorded spectra and represents the result without Compton suppression (i.e. the normal HPGe spectrum). The lower spectrum is with Comptonsuppression. The insert gives the ratio between the number of counts in the upper and lower spectrum, i.e. the Compton suppression as a function of energy. The loss of pulses in the photopeak due to random veto's is less than $2 \%$. The average Compton suppression between $100-1100 \mathrm{keV}$ amounts to 11.2 . The Compton edges are reduced to little bumps due to the small diameter of the entrance hole in the $\mathrm{NaI}(\mathrm{Tl})$ shield. Their shape differs appreciably from that of a normal peak. The single-escape peak of the $1332 \mathrm{keV}$ $\gamma$-ray is suppressed with the same factor as the background while the double-escape peak disappears due to the absence of collinear holes in the $\mathrm{NaI}(\mathrm{Tl})$ shield.

The areas of the 1173 and $1332 \mathrm{keV}$ peaks taken together amount to $65 \%$ of the total number of registered counts for the suppressed spectrum and $18 \%$ for the non-suppressed spectrum.

The Compton suppression for ${ }^{60} \mathrm{Co}$ with the 1.0 $\mathrm{mm}$ dead layer $\mathrm{Ge}(\mathrm{Li})$ crystal as the central detector is obtained similarly as described above. The result is shown in the middle part of fig. 6. The average Compton-suppression between 100 and $1100 \mathrm{keV}$ is now 8.4. The upper part of fig. 6 again shows the Compton suppression for the $0.2 \mathrm{~mm}$ dead layer HPGe.

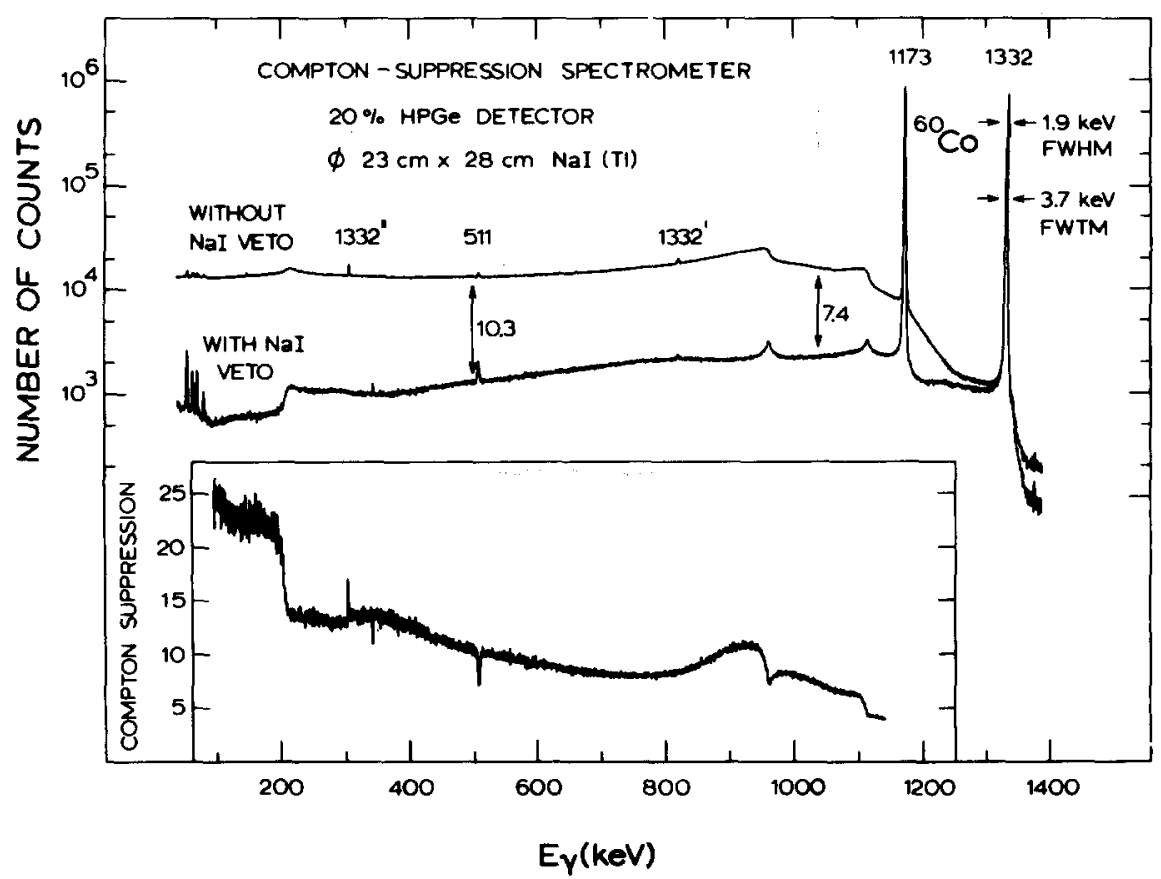

Fig. 5. Spectra of ${ }^{60} \mathrm{Co}$ for a $90 \mathrm{~cm}^{3}$ HPGe crystal as central detector. The upper spectrum is without Compton suppression, the lower spectrum is with Compton suppression. The insert shows the ratio between the number of counts in the upper and lower spectrum, i.e. the Compton suppression as a function of the energy. 

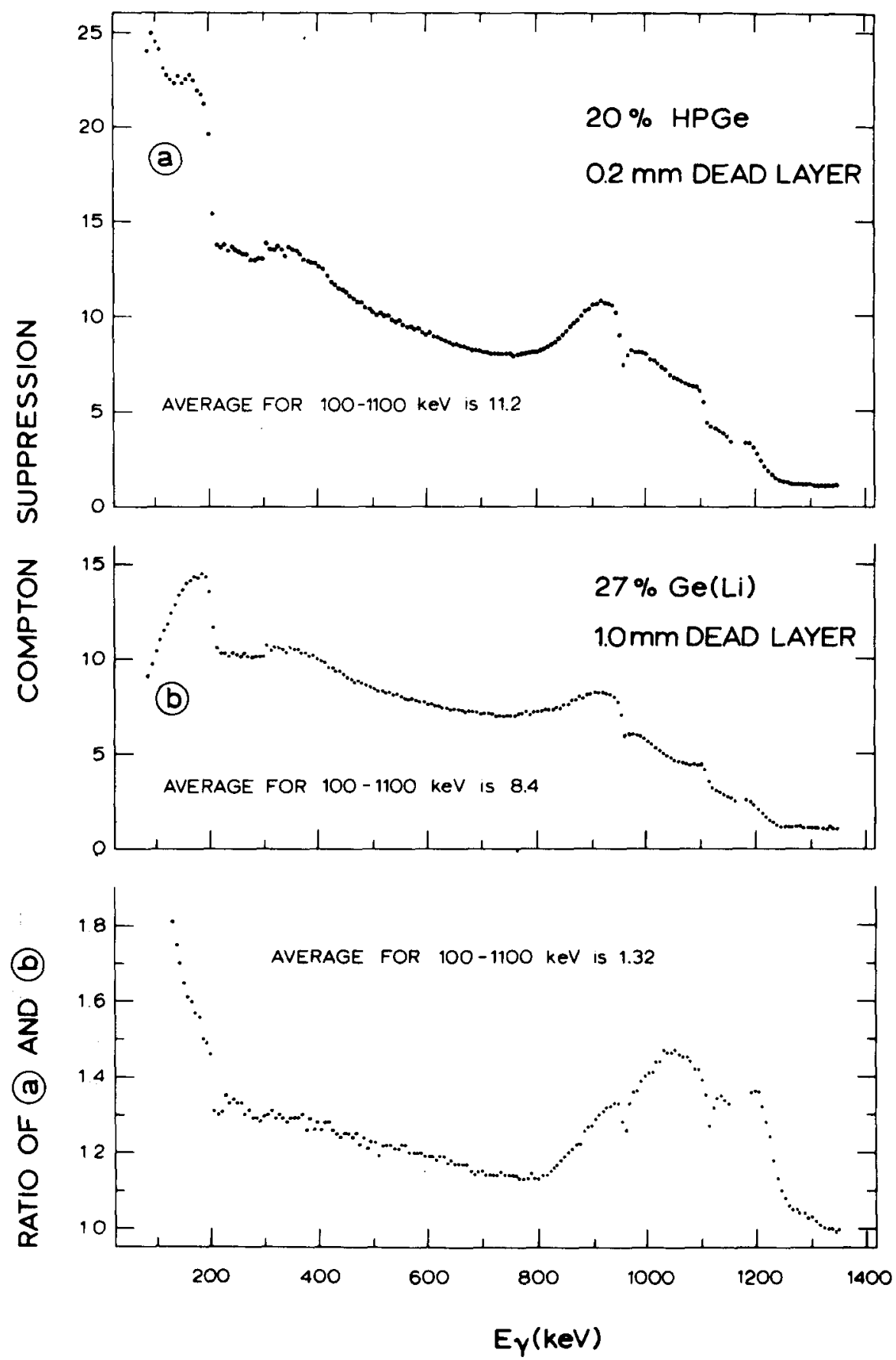

Fig. 6. Comparison of the Compton suppression for ${ }^{60} \mathrm{Co}$ for the $1.0 \mathrm{~mm}$ dead layer $\mathrm{Ge}(\mathrm{Li})$ and the $0.2 \mathrm{~mm}$ dead layer HPGe crystals as the central detector. The differences for $E_{\gamma}<200 \mathrm{keV}$ between the top and the middle curve are probably due to different timing properties.

The lower parts of fig. 6 gives the ratio of the two Compton suppressions and thus shows the improvement in performance of the spectrometer as a function of energy. The improvement is especially notable for the (high-background) region around the Compton edges (800-1150 keV), i.e. the energy 


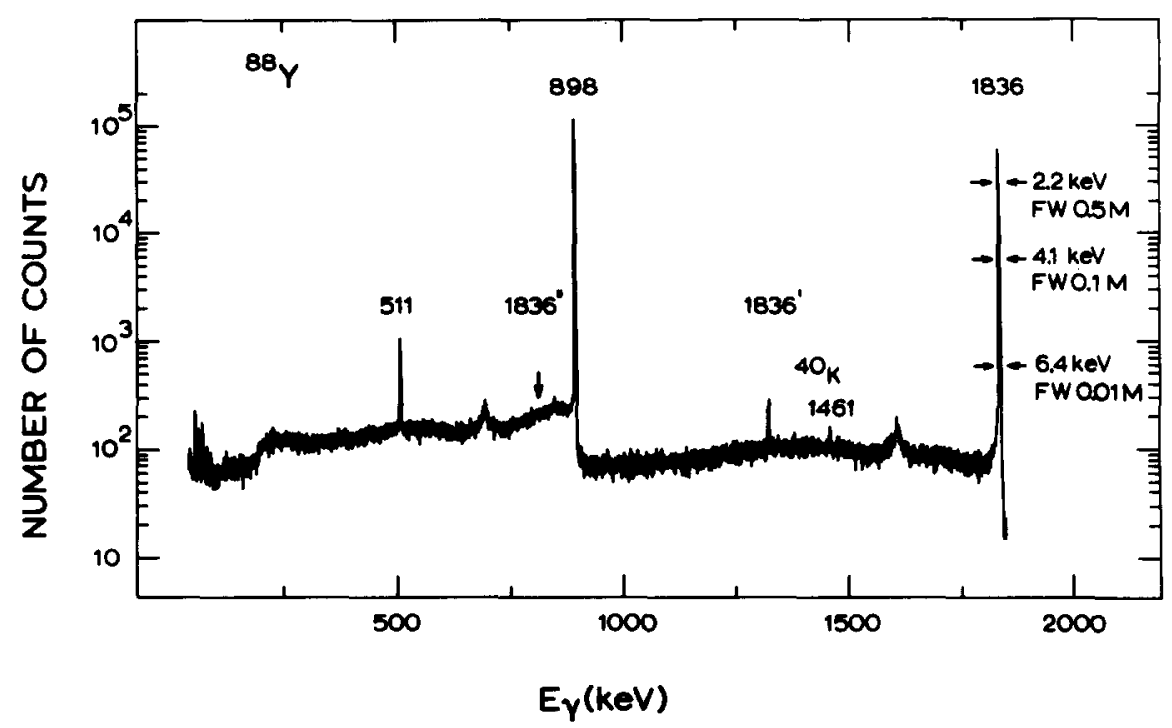

Fig. 7. Compton-suppressed spectrum of $\mathrm{a}^{88} \mathrm{Y}$ source.

region corresponding to the escape of Comptonscattered photons of low energy from the central detector. Reduction of the dead layer surrounding the central detector makes it more transparent for these low-energy $\gamma$-rays, which can now reach the surrounding shield more easily to produce a veto signal.

The average improvement for $100-1100 \mathrm{keV}$ is $32 \%$ in good agreement with the Monte Carlo prediction of $35 \%$.
3.3. Performance of the CSS with the thin-deadlayer HPGe as central detector for radioactive sources ${ }^{88} \mathrm{Y}$ and ${ }^{152} \mathrm{Eu}$ and the in-beam reaction ${ }^{24} \mathrm{Mg}+$ $(45 \mathrm{MeV})^{16} \mathrm{O}$

In fig. 7 we give the spectrum from a ${ }^{88} \mathrm{Y}$ source. The peak-to-Compton ratio for the $1836 \mathrm{keV}$ peak is 650 , i.e. the residual Compton background is lower by almost three orders of magnitude. The sum of the

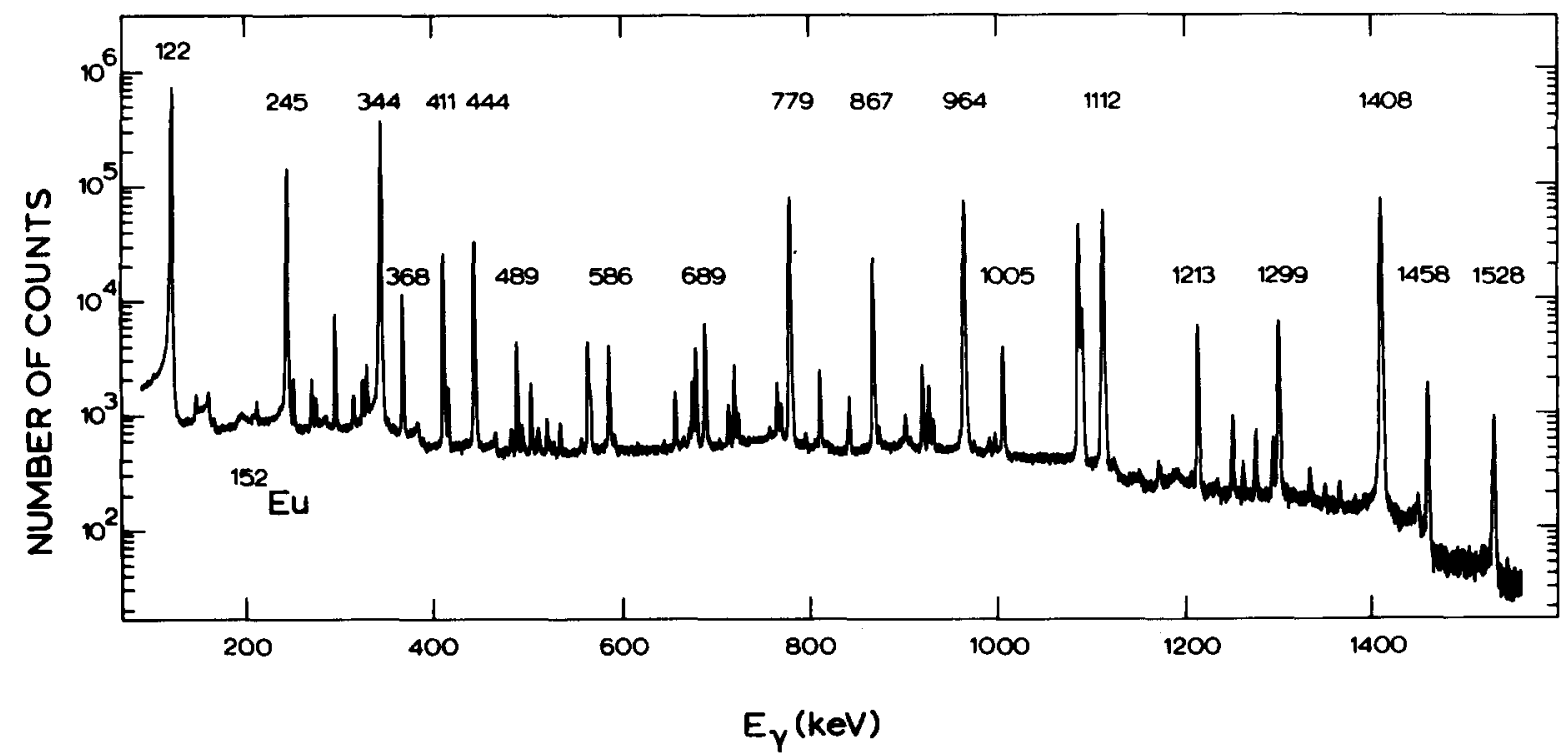

Fig. 8. Compton-suppressed spectrum of a ${ }^{152} \mathrm{Eu}$ source. 


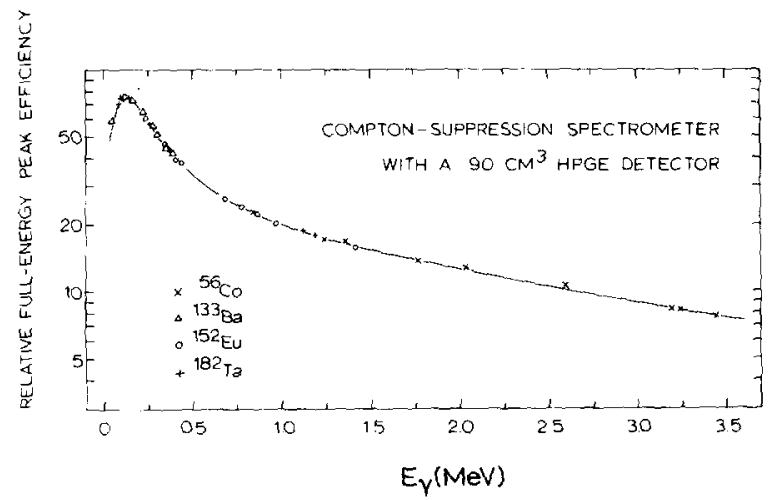

Fig. 9. The relative full-energy peak efficiency for the CSS with the $90 \mathrm{~cm}^{3}$ HPGe crystal as the central detector.

areas of the 898 and $1836 \mathrm{keV}$ peaks amount to $62 \%$ of the total number of registered counts.

In fig. 8 we show the more complicated $\gamma$-ray spectrum of a ${ }^{152} \mathrm{Eu}$ source. The relative full-energy peak efficiency for the CSS has been measured with
${ }^{56} \mathrm{Co}$ (ref. [5]), ${ }^{133} \mathrm{Ba}$ (ref. [4]), ${ }^{152} \mathrm{Eu}$ (ref. [5]) and ${ }^{182} \mathrm{Ta}$ (ref. [4]). The relative intensities are taken from the references indicated. The result is shown in fig. 9. The slope of the efficiency curve for higher energies is less steep, when compared with the HPGe detector outside the NaI shield. This reflects the thickness $(48 \mathrm{~mm})$ of the selectively irradiated part of the central detector (see fig. 1).

In fig. 10 we show the low-energy part $\left(E_{\gamma}<2\right.$ $\mathrm{MeV})$ of a singles $\gamma$-ray spectrum of the heavy-ion reaction ${ }^{24} \mathrm{Mg}+{ }^{16} \mathrm{O}$ at $E\left({ }^{16} \mathrm{O}\right)=45 \mathrm{MeV}$ and $\theta_{\gamma}=$ $90^{\circ}$. The measuring time was $5 \mathrm{~h}$ with a ${ }^{16} \mathrm{O}^{6+}$ beam current of $100 \mathrm{nA}$ (electrical) on a $320 \mu \mathrm{g} \cdot \mathrm{cm}^{-2}$, $99.94 \%$ enriched, ${ }^{24} \mathrm{Mg}$ target on thick $\mathrm{Au}$. The upper spectrum is without the Nal veto. The lower one is the Compton-suppressed spectrum. The suppression varies from about 7 to 12 .

This spectrum clearly shows the advantage of a CSS for in-beam $\gamma$-ray spectroscopy.

Weak and hardly visible peaks in the upper spectrum stand out clearly in the lower spectrum, see e.g. the 350 and $1400 \mathrm{keV}$ regions.

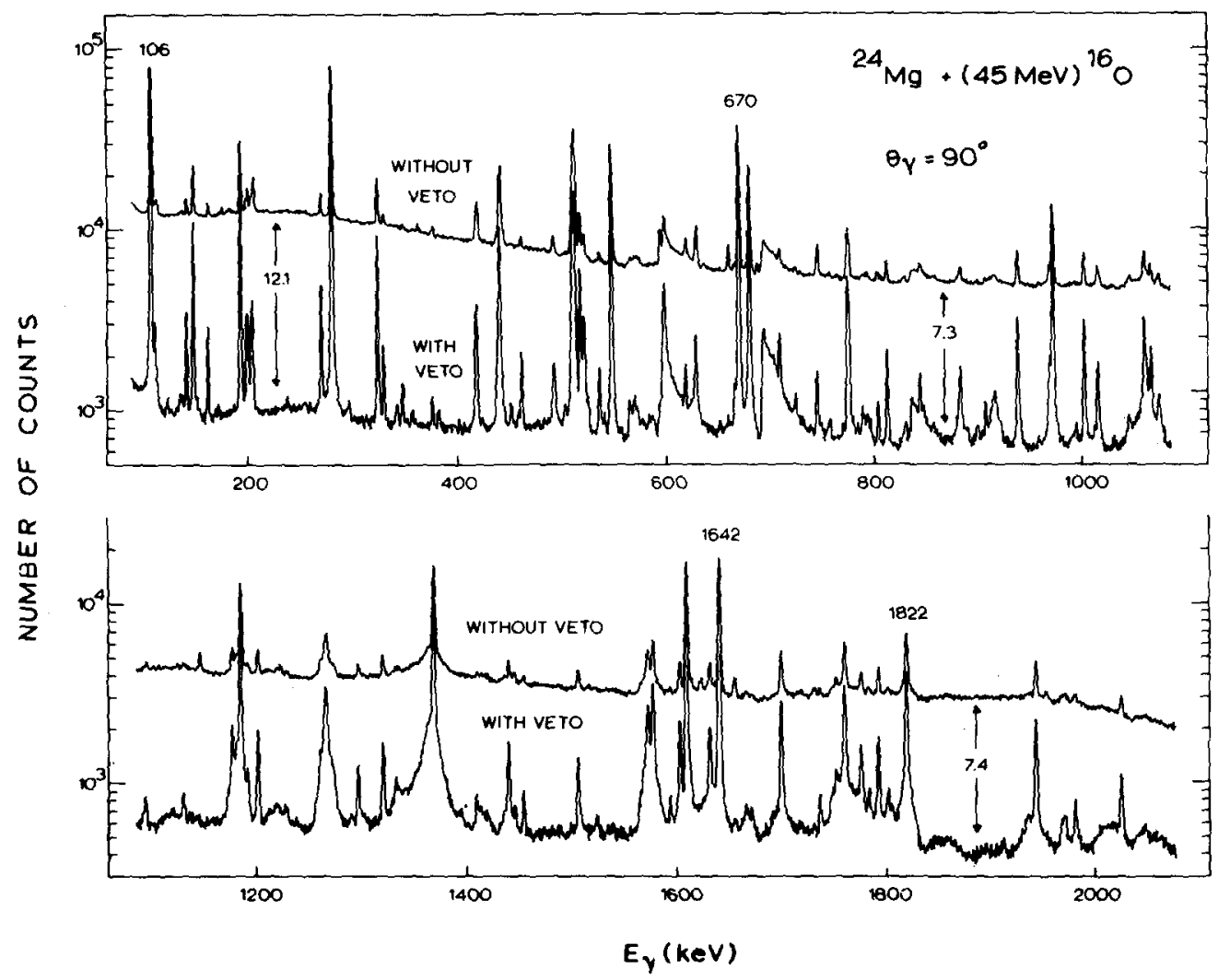

Fig. 10. Suppressed and non-suppressed $\gamma$-ray spectrum observed with the CSS at $\theta_{\gamma}=9.0^{\circ}$ in the bombardment of a $320 \mu \mathrm{g}$ $\mathrm{cm}^{-2}, 99.94 \%$ enriched, ${ }^{24} \mathrm{Mg}$ target on thick Au with a $45 \mathrm{MeV}, 100 \mathrm{nA},{ }^{16} \mathrm{O}^{6+}$ beam after $5 \mathrm{~h}$ measuring time. 